\title{
The Endocannabinoid System, Our Universal Regulator
}

\author{
Chad A. Sallaberry ${ }^{1 *}$, Laurie Astern
}

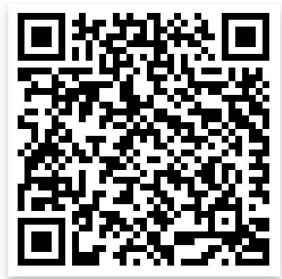

The endocannabinoid system (ECS) plays a very important role in the human body for our survival. This is due to its ability to play a critical role in maintaining the homeostasis of the human body, which encompasses the brain, endocrine, and immune system, to name a few. ECS is a unique system in multiple dimensions. To begin with, it is a retrograde system functioning post- to pre-synapse, allowing it to be a "master regulator" in the body. Secondly, it has a very wide scope of influence due to an abundance of cannabinoid receptors located anywhere from immune cells to neurons. Finally, cannabinoids are rapidly synthesized and degraded, so they do not stay in the body for very long in high amounts, possibly enabling cannabinoid therapy to be a safer alternative to opioids or benzodiazepines. This paper will discuss how ECS functions through the regulation of neurotransmitter function, apoptosis, mitochondrial function, and ion-gated channels. The practical applications of the ECS, as well as the avenues for diseases such as epilepsy, cancer, amyotrophic lateral sclerosis (ALS), and autism, which have no known cure as of now, will be explored.

\section{INTRODUCTION}

Despite various medical advances, there are still many more functions of the human body to uncover. Some of the less effective treatments lie within the field of mental health, due to the lack of accuracy and availability of tests for neurotransmitter function as well as apoptotic activity. The existing neurotransmitter tests utilize metabolites in urine (Hinz, Stein, Trachte, \& Ucini 2010); however, their applicability is currently very limited. We have not been able to show that the neurotransmitter levels measured in urine are as accurate as the actual levels in the central nervous system (CNS) or peripheral nervous system (PNS).

Apoptotic diseases such as cancer, acquired immune deficiency syndrome (AIDS), ALS, and autism, are all without an effective cure at the moment, and they seem to have similar pathology which involves neurotransmitter, mitochondrial, and apoptotic dysfunction (Favaloro, Allocati, Graziano, Di Lio, \& De Laurenzi 2012).

The ECS, unlike the CNS, PNS, and circulatory system, is one of the most understudied systems in the human body. It has been documented that ECS is directly involved with various roles in apoptosis, neurotransmitter levels, and homeostasis (Basavarajappa, Nixon, \& Arancio, 2009). ECS seems to carry a stigma because of the word "cannabis." In this paper, functions and possible benefits of ECS will be discussed.

\footnotetext{
${ }^{1}$ Department of Biochemistry and Molecular Biology, Colorado State University, 111 MRB Building, Fort Collins, CO 80523
}

\footnotetext{
*To whom correspondence should be addressed: csallaberry@bodylogicmd.com
}

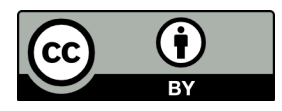

Except where otherwise noted, this work is licensed under https://creativecommons.org/licenses/by/4.0

doi:10.22186/jyi.34.5.48-55
The Endocannabinoid System Structure and Function

With all the complex cell signals, genetic mutations, and outside influences, how do we manage to stay at homeostasis? The answer is the endocannabinoid system. It is present nearly everywhere in the human body and functions by maintaining the homeostasis of the human body (Alger, 2013). This is achieved through a negative feedback loop which works by the activation of a postsynaptic neuron synthesizing and releasing the endocannabinoids as they target various cannabinoid $(\mathrm{CB})$ receptors.

These CB receptors are G-protein-coupled receptors (Gambi et al., 2005), which allow them to directly influence the incoming signals. This functions as an "override" signal, which differs from most other cells. As other cells have signal modifiers that can do anything from amplifying to diverging signals, the neuron is "over-riding" those cells. For example, a fracture in the toe would result in cell death. The resulting lymphatic response would increase blood flow and the migration of white blood cells to the surrounding areas. The ECS would then recognize the excess lymphatic signals, and after deciding that there is no longer a need for the increase of inflammation, the $\mathrm{CB}$ receptors in the surrounding immune cells and tissues will begin to bind with cannabinoids and start to slowly reduce these inflammatory responses.

A similar process occurs with pain signals in the brain. The binding and stimulation of $\mathrm{CB} 1$ receptors will upregulate the gamma-aminobutyric acid (GABA) neurotransmitters, thereby reducing pain signals throughout the brain. There are two main receptors in the ECS: the $\mathrm{CB} 1$ and $\mathrm{CB} 2$ receptors. $\mathrm{CB} 1$ receptors are located primarily within brain cells (including but not limited to the hippocampus, amygdala, and hypothalamus), and are not as densely expressed in the CNS, PNS, and the immune system. On the other hand, the CB2 receptors are located primarily in the CNS, PNS, immune system, and within white blood cells. Additionally, the existence of CB3 receptors is also hypothesized (Iqbal, 2007). These 
receptors will most likely be vast, with each one having a unique specialization despite being found in multiple locations throughout the body (Mazarnes, \& Carracosa 2006).

\section{The Endocannabinoids}

There are multiple known endocannabinoids that play a role in the ECS. All of them seem to play a role in anti-proliferative, anti-inflammatory, and anti-metastatic effects (Madia \& Daeninck, 2016). Additionally, it appears that they have a role in neurotransmitter, immune system, and mitochondrial function. There are two main endocannabinoids: anandamide and 2-archidonyl glycerol (2-AG).

Anandamide is an endocannabinoid in the human body. With the chemical formula $\mathrm{C}_{22} \mathrm{H}_{37} \mathrm{NO}_{2}$, it is referred to as the "bliss molecule." It can be released when one eats chocolate after a craving (Mackie, 2008). Anandamide may be a very important cannabinoid to manipulate for controlling pain stimuli. This is due to an interesting quality of anandamide in which the concentration of anandamide dictates the type and number of receptors activated. Anandamide also has the ability to make or break short-term connections between nerve cells that directly affect memory. There is speculation whether anandamide dulls and removes not only physical pain but psychological discomfort as well. If so, this could be utilized to help individuals with posttraumatic stress disorder (PTSD). This argument has particular merit as repression is a wellknown coping mechanism (De Petrocellis et al., 1998). Furthermore, anandamide has been shown to have anti-proliferative effects in breast cancer. It has also been shown to bind with a strong affinity to the $\mathrm{CB} 1$ receptors, which may play a greater role in the analgesic effects of the endocannabinoids.

2-Arachidonyl glycerol is the most prevalent endocannabinoid in the human body. Its chemical structure is quite similar to anandamide, having the same carbon backbone but a different $\mathrm{R}$ group, $\mathrm{C}_{23} \mathrm{H}_{38} \mathrm{O}_{4}$ (Gonsiorek, 2000). It is considered a full agonist of both the $\mathrm{CB} 1$ and $\mathrm{CB} 2$ receptors, playing a major role in ECS. Due to its high expression in peripheral immune cells, it seems to play a large role in anti-inflammation through immune suppression. Nonetheless, it also functions as a psychoactive endocannabinoid when it binds to CB1 receptors within brain cells.

\section{Cannabis}

Gertsch, Pertwee, and DiMarzo (2010) show that cannabis contains two very prevalent phyto-cannabinoids that target each $\mathrm{CB}$ receptor: tetrahydrocannabinol (THC) which is the active phytocannabinoid in cannabis primarily targeting the $\mathrm{CB} 1$ receptor, and $\beta$-caryophyllene (a terpene), which selectively targets the CB2 receptor (Prakash, Pandey, Amcaoglu, Venkatesh, \& Nagarkatti, 2009). These phyto-cannabinoids can mimic the action of endocannabinoids. However, it is hard to measure exactly how many of each of the $\mathrm{CB}$ receptors are being stimulated, and how much of each phyto-cannabinoid is entering the blood stream. However, since the cannabis plant can essentially function as a mass stimulation to the ECS, the body recognizes these phyto-cannabinoids as endocannabinoids.

\section{Lipid Metabolism}

The ECS runs through adipose tissue, demonstrating its role in adipogenesis, lipogenesis, and glucose uptake, all of which are stimulated by the $\mathrm{CB} 1$ receptor. Cannabinoids are unique in that they are rapidly synthesized as well as broken down soon after being used, which creates fewer long-term side effects. The two main enzymes that break down these endocannabinoids are fatty amide acid hydrolase (FAAH) and monoacylglycerol lipase (MAGL) (Petrosino \& Dimarzo, 2010). The endogenous cannabinoid system is extremely ubiquitous due to the fact that cannabinoids are both rapidly synthesized and degraded, which creates less longterm side effects.

Due to the previously stated ubiquitous nature, the effect of having the cannabinoid levels constantly altered for an extended period of time is not well known (Long et al., 2009). What is known is that FAAH is the preferred enzyme for the degradation of anandamide, whereas MAGL is the preferred enzyme for the degradation of 2-AG. Inhibitors of these enzymes have demonstrated success in stimulating the ECS. It is possible that by inhibiting one or both of these enzymes, the levels of various neurotransmitter could be adjusted and kept at a steady state for an extended period of time. This can be accomplished by preventing the hydrolysis of certain endo/phyto-cannabinoids that stimulate the release of various neurotransmitters.

\section{Apoptotic Pathway}

Apoptosis, which is the programmed death of cells, is an essential component of the cell cycle. This has multiple implications for the human body, such as maintaining the homeostasis or eradicating potentially dangerous cancer cells. While many of the proteins involved are known, the exact mechanisms of apoptosis are yet to be elucidated. Apoptosis is not the only manner in which cell death occurs; the other large contributor to cell death is necrosis (Elmore, 2007). However, unlike apoptosis, necrosis is highly toxic to cells and results in inflammation due to cellular lysis, which proceeds with cell death.

Apoptosis is generally caused by the activation of various caspases, a family of enzymes that play an essential role in apoptosis. Caspase-2, 8, 9, and 10 are considered initiators, whereas caspase-3, 6, and 7 are executioners. The three main pathways in which apoptosis can occur are extrinsic, intrinsic, and the perforin/ granzyme pathway.

In the extrinsic pathway, there is a clustering of receptors as well as the binding with their homologous ligand. For example, Fas ligand to receptor binding results in the adapter Fas-associated via death domain (FAA) and the binding of tumor necrosis factor (TNF) to its corresponding receptor. This then binds TNF receptorassociated death domain (TRADD; adapter protein) which associates with procaspase- 8 through dimerization of the death effector domain. This results in death-inducing signaling complex (DISC) which catalyzes the caspase- 8 . Caspase- 8 acts as an initiator which then triggers the execution phase.

On the other hand, the intrinsic pathway does not require external stimuli. Instead, it relies on intracellular stimuli produc- 
ing negative or positive signals. The signals can vary from loss of apoptotic suppression to loss of growth factors and toxins, among others. These stimuli cause changes in the mitochondria, resulting in mitochondrial permeability transition (MPT), loss of transmembrane potential, and the release of two main groups of apoptotic proteins, which then activate various caspases such as caspase-3 and 9.

The granzyme pathway is unique due to granzyme B's ability to cleave proteins at aspartate residues, resulting in direct activation of caspase-3, an executioner, thereby skipping the initiation phase of apoptosis. Additionally, this pathway plays a large role in T-cell- activated apoptosis. In a study conducted by Amcaoglu, Ashok, Ugra, Mitzi, and Prakash (2010), THC has been shown to cause cells to undergo enhanced and spontaneous apoptosis both in vitro and in vivo. Interestingly, mice treated with only THC had a higher rate of apoptosis than mice treated with both THC and mitogen (a substance that influences mitosis or cell division). Additionally, it was noted that active lymphocytes downregulated expression of $\mathrm{CB} 2$ receptors. However, CB1 agonists failed to have a significant impact in reducing THC-activated apoptosis, whereas CB2 agonists blocked the THC-induced apoptosis. This demonstrates the role of ECS in controlling various disease states and inflammation.

\section{Mitochondrial Function}

The mitochondria are responsible for converting carbohydrates and fatty acids into adenosine triphosphate (ATP) and providing energy for cells (Cooper, 2000). Uniquely, the mitochondria contain their own DNA. Similar to nuclear genomes, mutations can occur in mitochondrial genomes and cause disorders.

One of the main functions of ECS is modulating mitochondrial function. This can be accomplished through a variety of shared pathways. For example, a calcium-based pathway demonstrates how anandamide and 2-AG can modulate intracellular free calcium (Nunn, Guy, \& Bell, 2012). At low doses, anandamide produces expected result displaying anxiolytic (anti-anxiety) effects. However, at high doses, anandamide may activate transient receptor potential cation channel subfamily V member 1 (TRPV1), which would produce the opposite effect by upregulating mitochondrial function, thereby increasing anxiety. Anandamide has the ability to induce such effects due to its ability to bind with CB1 receptors at postsynaptic junctions, monitoring the opening of voltage-gated $\mathrm{Ca}^{2+}$ channels. This demonstrates that the modulation of calcium is more complicated than originally thought.

The ECS may also modulate mitochondria through redox reactions. Once again, this is another example of how ECS uses CB1 and $\mathrm{CB} 2$ receptors as a system of checks and balances within itself. This is evident due to the ability of CB1 receptors to increase reactive oxygen species (ROS), which results in an inflammatory cascade and mitochondrial stress. However, CB2 decreases ROS, resulting in the opposite effect. For example, an individual with a diagnosis of cancer may require an increase of mitochondrial stress to stimulate appetite, and modulation of both receptors through various agonists/antagonists might help control the activ- ity of the mitochondria. There are many other pathways, including the ceramide link and mechanistic target of rapamycin (mTOR) pathways that play a role in mitochondrial function which will be discussed later on in the paper.

An interesting fact regarding the mitochondria and their structure is the location of FAAH on the mitochondria, which is the primary fatty acid that degrades anandamide. This appears to be a strategic placement since anandamide plays a role in suppressing mitochondrial function. This placement allows the mitochondria to always be able to degrade anandamide if there was an excess of anandamide in the mitochondria. It would not be surprising to find that FAAH and MAGL (fatty acids) are located on various parts of the cellular infrastructure in which the ECS plays an important role in regulating. It cannot be emphasized enough how important the concentration is, as it pertains to their effect on mitochondrial function. Low concentrations of cannabinoids seem to benefit mitochondrial lifespan, function, ROS, and permeability, while it can cause serious damage to the mitochondria at higher concentrations.

Metabolic function and appetite have a direct association with mitochondrial function (Lipina, Irving, \& Hundal, 2014). Selectively blocking the $\mathrm{CB} 1$ receptor was shown to have a profound effect on appetite and metabolic function, which may be of help to obese individuals. However, it also causes incredible mood changes including anxiety and depression. This illustrates one of the most important points of ECS, which is its interconnectivity. ECS plays a role in the modulation of so many functions that in an attempt to change one, we may end up altering so many other functions that we cause more harm than good. It is not medically sound to block an entire cannabinoid receptor type; perhaps the focus should be on the individual pathways that are modulated by cannabinoid-receptor binding. For example, increasing FAAH located around the mitochondria could result in the degradation of anandamide, which has a negative impact on the mitochondria, while the remainder of anandamide can function separately and carry on its duties throughout the body.

\section{ECS and Mental Illness}

ECS is intriguing and relatively unexplored in terms of the role it plays in mental health. Manipulation of ECS might be beneficial in the treatment of schizophrenic patients. For example, in a clinical trial conducted with schizophrenic patients, anandamide levels were significantly higher in the blood of patients with acute schizophrenia when compared to healthy volunteers $(7.79 \pm 0.50$ vs. $2.58 \pm 0.28 \mathrm{pmol} / \mathrm{ml})$.

Anandamide is the second most common endocannabinoid and is extremely vital in the human body (De Marchi et al., 2003). The level of anandamide is an indicator of the acuity of the dysfunction of ECS as it relates to mental illness. Schizophrenia, as summarized by the National Institute for Mental Health (National Institute of Mental Health [NIMH], 2016b), is "a chronic and severe mental disorder that affects how a person thinks, feels, and behaves." People with schizophrenia may seem like they have lost touch with reality. Although schizophrenia is not as common as other mental disorders, the symptoms can be very disabling. 
One can conclude that the more severe the mental illness, the greater the dysfunction in the ECS. If we view mental illness on a continuum instead of separate entities, it would lend a more accurate view of mental illness. Some of the more common mental illnesses such as depression, anxiety, and schizophrenia, share similarities and generally contain overlapping symptoms when diagnosed. Sadness, suicidal thoughts, body dysmorphia, and panic attacks are the general symptoms of these conditions. The biggest difference is not the way someone feels, but the intensity of their feelings.

Based on this information, it would be logical to assume mental illnesses can be connected through a series of similar neurochemical imbalances and the resulting symptoms. It is important to note that some symptoms may intensify due to the positive or negative feedback loops as a result of the imbalances in various neurotransmitters, which give the appearance of a "new disease." For example, schizophrenic patients may appear to be solely depressed or anxious due to the mass dysfunction of neurotransmitters. These can then be amplified and may produce new symptoms such as hallucinations or extreme mania. However, that does not mean the chemical imbalances that cause depression or anxiety are gone; they are simply amplified, or in some instances, reduced. It appears that the different mental health illnesses may be interconnected in a web or network, which is maintained through neurotransmitter function. By testing and observing neurotransmitter function, one could obtain coordinates to see where in the web or network the issues with neurotransmitter are occurring. Errors in the ECS, which influence mood and perception, would help to explain these errors and malfunctions. Therefore, mental illness may be influenced by errors in the ECS as a result of miscommunication of the neurotransmitters. Since the ECS functions as a retrograde system, it can have a direct influence on the associated neurotransmitters. Therefore, targeting ECS might be a more effective treatment method than only addressing the neurotransmitters themselves. The current approach to addressing mental illness focuses on manipulating neurotransmitter release; however, this seems more like a band-aid treatment than an actual cure. Treating the underlying homeostatic issues through the ECS, thereby restoring neurotransmitter function, appears to be a more permanent solution.

\section{Current Treatments}

For the treatment of depression, the most common anti-depressants are selective serotonin reuptake inhibitors (SSRI) and other reuptake inhibitors (NIMH, 2016a). Various reuptake inhibitors function by preventing the reuptake of their specific neurotransmitters in the brain, allowing them to stay longer in the synapses and increase their concentrations. However, this is only a temporary fix that does not address the underlying cause of multiple neurotransmitter imbalances. Additionally, SSRIs only targets serotonin, yet most cases of depression are due to multiple neurotransmitter imbalances. Focusing solely on one neurotransmitter is not likely to result in improvement and could easily exacerbate the disease. The fact that SSRIs may increase depression and suicidal thoughts for some patients suggests that it may not be the best treatment option.

For anxiety, SSRIs are a common treatment; however, benzodiazepines are becoming the most prevalent form of treatment. The primary action of benzodiazepines is to bind to a pocket formed by GABA's alpha and gamma subunits (Griffin III, Kaye, Bueno, \& Kaye, 2013). This results in the conformational change in the GABA-A receptor, which induces the inhibitory effect of GABA. GABA is highly concentrated in the limbic system, which is the system most closely associated with addiction. This is one of the most prevalent issues with benzodiazepines, as focusing solely on GABA increases the chance for addiction. Benzodiazepine's short half-life contributes to the extremely addictive nature of the drug. Benzodiazepines are listed as a schedule IV drug (United States Drug Enforcement Administration, n.d.), which implies that along with its ability to treat disease, it has a relatively low potential for abuse. However, this is not the case. According to the National Institute on Drug Abuse (2017), there were approximately 8,700 overdose-related deaths in 2015, which was a 4.3 -fold increase from 2002.

\section{New Directions}

As stated above, there are clear concerns about the way mental illnesses are being treated. One of the largest concerns is related to a lack of neurotransmitter testing to determine the contributions of various neurotransmitters to mental illness. From there, future treatments should address the underlying causes without the utilization of addictive substances.

GABA has a unique relationship with the ECS (Sigel et al., 2011). When 2-AG is activated, it enhances the effects of GABA by causing an increase of the GABA neurotransmitters in the human body (Manzanares \& Carracosa, 2006). However, when the $\mathrm{CB} 1$ receptor is activated by endocannabinoids or phyto-cannabinoids, it inhibits GABA. This is not surprising as the ECS is responsible for a majority of the body's homeostatic functions. If one were to activate the body's ECS by introducing an influx of endocannabinoids or phyto-cannabinoids, and predetermine which receptors were activated, it could give us bi-directional control over GABA that current drugs fail to offer.

For example, to increase an individual's GABA levels, one could take a CB1 inhibitor such as rimonabant, which prevents $\mathrm{CB} 1$ activation. This would result in 2-AG becoming the primary endocannabinoid synthesized due to 2-AG's affinity to the CB2 receptor, subsequently increasing GABA concentration. To decrease GABA, one could utilize an agonist of MAGL to increase degradation of 2-AG. This would result in having a stable level of anandamide concentration with a much lower 2-AG concentration.

Treatments that would directly affect the ECS might be more beneficial than medication currently used to treat mental illnesses. This is due to the ability of cannabinoids to correct the neurochemical imbalances by attacking the source of the issue, rather than just attempting to alleviate the symptoms.

Apoptotic Functions and Potential Cures for Cancer, ALS, HIV, Autism, and Epilepsy

$H I V$ 
HIV is generally caused by increased apoptosis of primarily CD4+ and CD8+ T-cells. Initially, HIV stimulates various cellular cascades that eventually leads to permanent conformational changes (Simon, Ho, \& Karim, 2006). Not only is apoptosis increased in HIV glycoproteins, but cellular activation is increased as well, resulting in an increase in turnover of T-cells. This seems to indicate an increase of T-cell destruction rather than a lack of T-cell production.

T-cells are very important for the immune response against pathogens, extracellular parasites, and other possible viral or bacterial organisms (Zhu \& William, 2008). Unlike cancer, apoptosis is not suppressed, but rather excited in the presence of HIV. However, one of the incredible mechanisms regarding ECS is that even though it is considered a "negative feedback loop" as a result of varying receptors and cannabinoids, it appears that it is possible to both decrease and increase apoptosis. Hypothetically, if one were able to reduce T-cell apoptosis which occurs in the early stage of HIV, one might be able to reverse the process completely and eradicate the virus.

Cancer

Cancer is a result of multiple genetic mutations that allow cells to multiply uncontrollably, evade apoptosis, become immortal, and undergo metastasis. Some of the genes are proto-oncogenes, such as Ras (Lodish, Berk, \& Zipursky, 2000), while others are tumor suppressor genes (TSG) (Zhu, et al, 2015), such as BRCA1 and BRCA2 (Lowe \& Lin, 2000). p53 is another TSG, which also plays an extremely important role as a cell-cycle checkpoint protein involving cycle arrest.

Common forms of cancer treatment include radiotherapy, which blasts cancer cells with radiation in hopes of eradicating all of the cancer cells, is not a sustainable treatment. There are other pharmacological interventions. For example, Gleevec (imatinib mesylate) has been shown to be especially effective (RX list, 2017) as an anti-cancer treatment. Gleevec functions as a small molecule kinase inhibitor. As the action of Gleevec is targeted, it is a much safer approach. However, this renders its use very selective, such that it has a high success rate only if the Philadelphia chromosomal abnormality is present (Pray, 2008). An additional problem with drugs such as Gleevec is their effect on the p-glycoprotein (Schinkel, 1999), which functions as a "selective pump" in the blood-brain barrier and other various sanctuaries in the body. However, this function backfires with various anti-cancer drugs, as it tends to pump them out in an attempt to detoxify the body.

The ECS should be evaluated as a possible alternative to treat cancer. One of the keys to eradicating cancer is the selective induction of apoptosis. THC has been shown to trigger apoptosis, as mentioned earlier. If we could determine the appropriate cannabinoids (THC, THCA, CBD, 2-AG, etc.) associated with cancer, we could take a step-wise approach to neutralize specific cancers. This would be accomplished by injecting the specific cannabinoids into the cancerous tumor via a virus or vector. Since CB2 has the largest effect on apoptosis, we could enhance CB2 binding by utilizing an antagonist of MAGL. This would prevent the cannabinoids that generally bind to the CB2 receptor from being degraded, resulting in an increased and prolonged concentration of cannabinoids within the blood stream.

The specific fatty acids to be blocked or enhanced may vary from cancer to cancer, as would the cannabinoids used in treatment. This may be an effective way to induce apoptosis inside the cell, which would eradicate cancer while minimizing adverse reactions commonly associated with conventional chemotherapy. This could prove to be very successful due to the fact that once certain mutations in the cell have resulted in cancer, the ability to process a normal signal from p53 and other TSG/oncogenes is lost. Through utilizing the ECS to override these cancerous cells, these cells may be induced to attend to the new apoptotic signals.

Autism

Autism, or autism spectrum disorder (ASD), is a disease which involves both physical and behavioral changes. The cells in the CNS which are most affected are the GABAergic Purkinje neurons (Goodenowe \& Pastural, 2011). These neurons are the only output of the cerebellar cortex and play a vital role in the function and design of the cerebellum circuits. The loss of these specific GABAergic neurons is one of the primary causes of autism. While apoptosis gone awry could be the source of the disappearance of these Purkinje neurons, this is typically not the case. The most common catalysts which are responsible for their destruction are alcohol and other toxins (Sudarov, 2013). However, since the neurons are GABAergic, the ECS would not be able to replicate these particular neurons, but manipulation of the system could allow us to modulate the GABA signals that should occur within the deceased Purkinje neurons.

Another indicator of autism is mass mitochondrial dysfunction, which can be detected in numerous ways, including but not limited to: plasma lactate levels, carnitine levels, and glutathione levels (Goodenowe \& Pastural, 2011). Interestingly, the Purkinje neuron has been associated with certain mitochondrial disorders that signal autism. Excessive amounts of glutamate formed by microglia can form around Purkinje neurons. This can be caused by presynaptic depolarization of climbing fiber neurons, which play a role in massive action potential spikes (Ohtsuki, Piochon, \& Hansel, 2009) and synaptic spillover, which can result in glutamate toxicity.

Additionally, there is an interesting gender bias in autism, with prevalence nearly four times as much in prepubescent males than in prepubescent females. This is due to a four-fold increase of estrogen in females, which seems to function as a protective mechanism for mitochondria, especially B-estradiol. It is therefore not surprising that the mitochondrial malfunctions that are protected best by B-estradiol are related to glutamate toxicity.

In addition to their role with the GABA/glutamate neurotransmitters, the ECS has also been shown to play a direct role in controlling mitochondrial malfunction. As previously mentioned, the ECS is known to modulate many pathways, including $\mathrm{Ca}^{2+}$ channels, Kir, MAPK, e/iNOS, mTor, and ceramide production. This demonstrates that the ECS has a firm "hold" over mitochondrial 
production (Nunn, Guy, \& Bell, 2012). Through these different pathways, various endocannabinoids are expressed, exhibiting a system of diverse responses within these pathways. These range from increasing/decreasing apoptosis, increasing nNOD in neurons, and decreasing iNOS in astrocytes.

There is also a plethora of other controls within these pathways which are just as important with regards to mitochondrial production. However, one of the most important pathways is mTOR, which controls mitochondrial function and lifespan. Although the ECS is generally considered anti-proliferative, throughout the mTOR pathway (specifically CB2 activation), it has been demonstrated that the ECS can become proliferative, solely through low-level activation. This activation might provide treatment for mitochondrial dysfunction in autism as a result of the ECS's mass control over the mitochondrial pathways.

A key to altering mitochondrial function in patients with autism could be FAAH, which is the primary enzyme responsible for degrading anandamide and THC (National Institute of Neurological Disorders and Stroke, 2013). FAAH inhibitors have been known to show analgesic, anti-inflammatory, and anti-depressant properties in mice. This makes sense since with FAAH lowered or removed, the endogenous cannabinoid anandamide would be more prevalent in the bloodstream, and it has been known to produce the aforementioned effects through interactions with various parts of the brain and immune cells. This could be a possible course of treatment in individuals with autism, since their mitochondria may be downregulated. Inhibiting FAAH would allow for prolonged interaction with anandamide, which at the right concentration, will upregulate mitochondrial function.

Amyotrophic Lateral Sclerosis (ALS)

ALS is a neurodegenerative disease that can affect nerve cells in the brain and spinal cord (specifically motor neurons), resulting in their death. Consequently, the brain loses the ability to control muscles, including those of lung and heart (Ahn, Johnson, \& Benjamin, 2009). ALS is only understood up to a point, with multiple theories in agreement that cell death contributes to this process. One of the more salient theories is that apoptosis plays a large role in ALS, and is responsible for the death of motor neurons (Sathasivam, Ince, \& Shaw, 2001). It has been reported that in ALS, there are changes in p53 proteins and the Bcl-2 family.

Cancer is another disease that exhibits mutations similar to these proteins; however, opposite effects take place. In cancer, there is uncontrolled cell growth, which contrasts with the uncontrolled cell death found in ALS. However, the cell death in ALS specifically targets the motor neurons. In a study conducted by Ranganathan and Bowser (2010), p53 levels were found elevated significantly in the spinal cord, but not so in the motor neurons. This finding might partially explain the death of motor neurons in ALS, and suggests that ALS may be a two-tiered disease.

Another presenting issue in most ALS patients is their glutamate levels (Foran \& Trotti, 2009). Glutamate excitotoxicity is very common in neurodegenerative diseases such as ALS. Various glutamate transporters known as the excitatory amino acid trans- porter (EAAT) family have a large role in glutamate regulation, although structural differences exist. EEAT-2 appears to be important in glutamate transport due to its abundance in the brain and within the CNS. In postmortem studies of ALS patients, there is a clear downregulation of EEAT-2 present within the ventral horn of the spinal cord, demonstrating the presence of glutamate excitotoxicity.

Excitotoxicity is modulated primarily through the $\mathrm{Ca}^{2+}$ pathways, which was discussed in the section on autism, and is influenced by the ECS. So, it is not a surprise that glutamate is one of the neurotransmitters that the ECS influences. ALS appears to be the result of apoptosis and abnormalities with glutamate transport. It seems that one may act as a catalyst for the other. It is possible that the increase of apoptotic activity in caspases- 8 (initiator) and 3 (executioner) can lead to the increased apoptosis of motor neurons. This phenomenon then results in a dysregulation of glutamate transport, subsequently causing excitotoxicity which can be the primary factor in morphing ALS into a deadly disease.

Our hypothesis is that untreated ALS has an exponential growth curve, despite not being apparent in the review conducted. Once a certain percentage of motor neurons have died, there is an increase in dysregulation of proteins (such as EEAT-2), which result in the inevitable death of the ALS patients. We believe that downregulation of apoptosis through the ECS will at some point lead to an eventual cure. Another hypothesis is that some other potential pathways may reduce the emission of glutamate, even after the downregulation of various proteins.

Epilepsy

Epilepsy is a complex disease in which there are multiple theorized causes, such as epigenetics, neurotransmitter imbalance, iongated channel dysfunction, etc. However, we will be focusing on the previously mentioned variables and their effect on epilepsy.

Epileptic seizures are not all the same (Bromfield, Cavazos, \& Sirven, 2006); there are partial seizures and generalized seizures. The main cause of epilepsy is the failure of the membrane to reach its equilibrium post-action potential (Bromfield et al., 2006). Action potentials are formed due to depolarization of the neuronal membrane, which prompts the release of neurotransmitters at the axon terminal. These are brought about by net positive changes in various ion fluxes such as the ligand or voltage-gated channels, or changes in intracellular ion compartmentalization. There are eight types of neurotransmitters in the brain; however, we will be focusing primarily on the major excitatory and inhibitory neurotransmitters, glutamate and GABA, respectively.

Neuronal excitability has many variables that can determine how large the excitatory and inhibitory effect can be, including the modulation of gene expression, type or number of gated channels, and changes in extracellular ion concentrations. However, we notice that a lot of these are modulated by the homeostatic functions of the ECS (Rosenberg, Tsien, Whalley, \& Devinsky, 2015). There is a clear link between cannabis and its anticonvulsant properties, but it is important to note the difference between the exogenous cannabinoids in cannabis and the endogenous cannabinoids that 
the body produces (Alger, 2014). For example, it makes sense that the exogenous/phyto-cannabinoids have a much broader effect on our body. This effect can be referred as the "generalized cannabinoid arc." When smoking marijuana, regardless of the strain, there are a series of effects that generally occur. This could be due to the fact that, although they mimic the endogenous cannabinoids in our body, they were not designed to modulate our various systems.

Since the cannabinoids are endogenous in nature, we would argue that every endocannabinoid and $\mathrm{CB}$ receptor play a specific role in the homeostatic function of the muscle, organelle, or cascade that it controls. So, despite the success thus far that has been achieved by treating epilepsy with cannabis, perhaps a more optimal treatment route would be to utilize the ECS directly. Despite the challenges, we believe that it would provide a more effective treatment for epilepsy. This raises the question of how we use the intricate pathways of ECS to manipulate the neurotransmitters and action potentials. Interestingly, the most efficient way to stimulate short-term endothelial CB (eCB) mobilization would be utilizing depolarizations of postsynaptic membranes that last from $1 \mathrm{~s}$ to 10s. Action potentials are also depolarizations, specifically involving the neuronal membrane. If artificial stimulation of $\mathrm{eCB}$ is a similar process to these action potentials, it would make sense that the body's natural response to these action potentials is to control them with ECS due to the homeostatic function of the systems.

One could hypothesize that an error within ECS could result in epilepsy. The most promising treatment for epilepsy may be utilizing the CB1 receptors located throughout the brain, specifically, for their response to action potentials and their ability to manipulate GABA in epilepsy. Regardless of whether the miscommunication in the ECS is the cause or simply a side effect of epilepsy, a possible path to curing it is through manipulating the ECS with a prolonged cannabinoid therapy. To achieve this, we must direct research into the specificity of each endocannabinoid and their respective receptors. Once we fully decode the ECS and its role with respect to epilepsy, we would be able to not only stop seizures, but also, through extended therapy, correct the errors causing the prolonged firing of action potentials which induce the occurrence of seizures.

\section{CONCLUSION}

The ECS is one of the, if not the most, important systems in our body. Its role in the homeostatic function of our body is undeniable, and its sphere of influence is incredible. Additionally, it also plays a major role in apoptotic diseases, mitochondrial function, and brain function.

Its contribution is more than maintaining homeostasis; it also has a profound ability in regulation. Working in a retrograde fashion and with a generally inhibitory nature, ECS can act as a "kill switch." However, it has been shown to play an inhibitory or stimulatory role based on the size of the influx of cannabinoids, resulting in a bimodal regulation. Furthermore, due to the nature of the rate of degradation of cannabinoids, it does not have as many long-term side effects as most of the current drugs on the market.
The ECS may not only provide answers for diseases with no known cures, but it could change the way we approach medicine. This system would allow us to change our focus from invasive pharmacological interventions (i.e. SSRIs for depression, benzodiazepines for anxiety, chemotherapies for cancer) to uncovering the mystery of why the body is failing to maintain homeostasis. Understanding the roles of ECS in these diseases confers a new direction for medicine which may eradicate the use of some of the less tolerable therapeutics.

\section{REFERENCES}

Ahn, K., Johnson, D., \& Cravatt, B. (2009). Fatty acid amide hydrolase as a potential therapeutic target for the treatment of pain and CNS disorders. Expert Opinions on Drug Discovery, 4(7), 763-764. doi: $10.1517 \% 2 \mathrm{~F} 17460440903018857$

Alger, B. (2013). Getting High on the endocannabinoid system. Cerebrum: The Dana Forum on Brain Science, 14.

Alger, B. E. (2014). Seizing an Opportunity for the Endocannabinoid System. Epilepsy Currents, 14(5), 272-276. doi:10.5698/1535-7597-14.5.272

Amcaoglu, R., Ashok, C., Ugra, S., Mitzi, N., \& Prakash, N. (2010). Cannabinoidinduced apoptosis in immune cells as a pathway to immunosuppression. Immunobiology, 215(8), 598-605. doi:10.1016/j.imbio.2009.04.001

Basavarajappa, B., Nixon, R., \& Arancio, O. (2009). Endocannabinoid System: Emerging Role from Neurodevelopment to Neurodegeneration. Mini-Re views in Medicinal Chemistry. 9(4). doi:10.2174/138955709787847921

Bromfield, E. B., Cavazos, J. E., \& Sirven, J. I. (2006). Basic Mechanisms Underlying Seizures and Epilepsy. An Introduction to Epilepsy. West Hartford (CT): American Epilepsy Society.

Bromfield, E. B., Cavazos, J. E., \& Sirven, J. I. (2006). Clinical Epilepsy. An Introduction to Epilepsy. West Hartford (CT): American Epilepsy Society.

Cooper, G. M. (2000). The Cell: A molecular Approach. Sunderland (Mass), Sinauer Associates.

De Marchi, N., De Petrocellis, L., Pierangelo, O., Fabiana, D., Filomena, F., \& Di Marzo, V. (2003). Endocannabinoid signaling in the blood of patients with schizophrenia. Lipids in Health and Disease, 2(5). doi:10.1186/1476511X-2-5

De Petrocellis, L., Palmisano, A., Melck, D., Bissogno, T., Laezza, C., Bifuclo, M., \& Di Marzo, V. (1998). The endogenous cannabinoid anadamide inhibits human breast cancer cell proliferation. Proceedings National Academy of Science USA, 95(14), 8375-8380

Elmore, S. (2007). Apoptosis: A review of Programmed cell death. Toxicology Pa thology, 35(4), 495-516. doi:10.1080/01926230701320337

Favaloro, B., Allocati, N., Graziano V., Di lio, C., \& De Laurenzi. (2012). Role of Apoptosis in disease. Aging, 4(5), 330-349. doi:10.18632/aging.100459

Foran, E., \& Trotti, D. (2009). Glutamate transporters and the excitotoxic path to motor neuron degeneration in amyotrophic lateral sclerosis. Antioxidants and Redox signaling, 11(7), 1587-1602. doi:10.1089/ars.2009.2444

Gambi, F., De Beradis, D., Sepede, G., Quartesan, R., Calcagni, E., Salerno, R. M., ... Ferro, F. M. (2005). Cannabinoid receptors and their relationships with neuropsychiatric disorders. International Journal Immunopathology Pharmacology, 18(1), 15-9. doi:10.1177/039463200501800103

Gertsch, J., Pertwee, R., \& DiMarzo, V. (2010). Phyto-cannabinoids beyond the Cannabis plant-do they exist? British Journal of Pharmacology, 160(3), 523 529. doi:10.1111/j.1476-5381.2010.00745.x

Gonsiorek, W., Lunn, C., Fan, X., Narula, S., Lundell, D., \& Hipkin, R. W. (2000). Endocannabinoid 2-arachidonyl glycerol is a full agonist through human type 2 cannabinoid receptor: antagonism by anandamide. Molecular pharmacology, 57(5), 1045-50.

Goodenowe, D., \& Pastural, E. (2011). The Biochemical Basis of Autistic Behavior and Pathology. Autism - A Neurodevelopmental Journey from Genes to Behaviour. Rijeka: Intech. doi:10.5772/18571

Griffin III, C., Kaye, A., Bueno, F., \& Kaye, A. (2013). Benzodiazeprine Pharmacology and Central Nervous system-mediated effects. Ochsner Journal, 13(2), 214-223. 
Hinz, M., Stein, A., Trachte G., \& Uncini, T. (2010). Neurotransmitter testing of the urine: a comprehensive analysis. Dove Press, 2010(2), 177-183. doi:10.2147/RRU.S13370

Lipina, C., Irving, A., \& Hundal, H. (2014). Mitochondria: a possible nexus for the regulation of energy homeostasis by the endocannabinoid system? American Journal of Physiology-Endocrinology and Metabolism, 307(1), 1-13. doi:10.1152/ajpendo.00100.2014

Lodish, H., Berk, A., \& Zipursky, S. L. (2000). Proto-onco genes and tumor-supressor genes. Molecular Cell biology. New York: W.H. Freeman.

Long, J., Normura, D., Vann, R., Walentiny, M., Booker, L., Jin X., ... Cravatt, B. (2009). Dual blockade of FAAh and MAGL identifies behavioral processes regulated by endocannabinoid crosstalk in vivo. Proceedings of the $\mathrm{Na}$ tional Academy of Sciences of the United States of America, 106(48). doi:10.1073/pnas.0909411106

Lowe, S., \& Lin, A. (2000). Apoptosis in Cancer. Carcinogenesis. Oxford Academic Journal, 21(3), 485-495.

Mackie, K. (2008). Cannabinoid receptors: where they are and what they do. Journal of Neuroendocrinology, 20, 10-14. doi:10.1111/j.1365-2826.2008.01671.x

Maida, V., \& Daeninck, P. J. (2016). A user's guide to cannabinoid therapies in oncology. Current Oncology. 23(6), 398-406. doi:10.3747/co.23.3487

Manzanares, J., \& Carrascosa, A. (2006). Role of the cannabinoid system in pain control and therapeutic implications for the management of acute and chronic pain episodes. Current Neuropharmacology, 4(3), 239-257.

Manzanares, J., Julian, M., \& Carracosa, A. (2006). Role of the Cannabinoid system in pain control and therapeutic implications for the management of acute and chronic pain episodes. Current neuropharmacology. 4(3), 239-257.

National Institute on Drug Abuse. (2017). Overdose Death Rates. Retrieved from https://www.drugabuse.gov/related-topics/trends-statistics/overdose-deathrates

National Institute of Mental Health. (2016a). Mental Health Medications. Re trieved from: https://www.nimh.nih.gov/health/topics/mental-health-medications/index.shtml

National Institute of Mental Health. (2016b). Schizophrenia. Retrieved from: https://www.nimh.nih.gov/health/topics/schizophrenia/index.shtml

National Institute of Neurological Disorders and Stroke. (2013). Amyotrophic Lateral Sclerosis (ALS) fact sheet. Retrieved from: https://www.ninds.nih.gov/Disorders/Patient-Caregiver-Education/Fact-Sheets/Amyotrophic-LateralSclerosis-ALS-Fact-Sheet

Nunn, A., Guy, G., \& Bell, J. (2012). Endocannabinoids in neuroendopsychology: Multiphasic control of mitochondrial function. Philosophical Transactions B, 367(1607), 3342-3352. doi:10.1098/rstb.2011.0393

Ohtsuki, G., Piochon, C., \& Hansel, C. (2009). Climbing Fiber Signaling and Cerebellar Gain Control. Frontiers in Cellular Neuroscience, 3, 4. doi:10.3389/neuro.03.004.2009

Omar, I. (2007). Endocannabinoid system pathophysiology of apidogenesis: current management of obesity. Personalized Medicine, 4(3), 307-319.

Petrosino, S., \& Di Marzo, V. (2010). FAAH and MAGL inhibitors: therapeutic opportunities from regulating endocannabinoid levels. Current Opinion in Investigational Drugs, 11(1), 51-62.

Prakash, N., Pandey, R., Amcaoglu, R., Venkatesh, H., \& Nagarkatti, M. (2009). Cannabinoids as novel anti-inflammatory drugs. Future Medicinal Chemistry, 1(7), 1333-1349. doi:10.4155/fmc.09.93

Pray, L. (2008). Gleevec: The Breakthrough in Cancer Treatment. Nature Education, 1(1), 37.

Ranganathan, S., \& Bowser, R. (2010). p53 and Cell Cycle Protiens Participate in Spinal Motor Neuron Cell Death in ALS. The Open Pathology Journal, 4, 11-22. doi:10.2174/1874375701004010011

Rosenberg, E. C., Tsien, R. W., Whalley, B. J., \& Devinsky, O. (2015). Cannabinoids and Epilepsy. Neurotheraputics, 12(4), 747-768. doi:10.1007/s13311015-0375-5

RX List. (2017). Gleevec. Retrieved from: http://www.rxlist.com/gleevec-drug.htm

Sathasivam, S., Ince, P. G., \& Shaw, P. J. (2001). Apoptosis in amyotrophic lateral sclerosis: a review of the evidence. Neuropathology and Applied Neurobiology, 27(4), 257-74.

Schinkel, A. H. (1999). P-Glycoprotien, a gatekeeper in the blood brain barrier Advanced Drug Delivery Reviews, 36(2-3), 179-194.
Sigel, E., Baur, R., Racz, I., Smart, T. G., Zimmer, A., \& Gertsch, J. (2011). The major central endocannabinoid directly acts at GABA(A) receptors. Proceedings of the National Academy of Science, 108(44), 18150-18155. doi:10.1073/ pnas. 1113444108

Simon, V., Ho, D., \& Karim, Q. (2006). HIV/AIDS epidomology pathogensis, prevention, and treatment. Lancet, 368(9534), 489-504.

Sudarov, A. (2013). Defining the role of Cerebellar Purkinje cells in autism spectrum disorders. Cerebellum. 12(6), 950-95. doi:10.1007/s12311-013-0490-y

United States Drug Enforcement Administration. (n.d.). Drug Scheduling. Retrieved from https://www.dea.gov/druginfo/ds.shtml

Zhu, J., \& William, P. (2008). CD4 T cells: fates, functions, and faults. Blood, 112(5), 1557-1569. doi:10.1182/blood-2008-05-078154

Zhu, K., Liu, Q., Zhou, Y., Tao, C., Zhongming, Z., Sun, J., \& Xu, H. (2015). Oncogenes and tumor suppressor genes: comparative genomics and network perspectives. BMC Genomics, 6(7). doi:10.1186/1471-2164-16-S7-S8 